\title{
ANATOMIA ECOLÓGICA DOS ELEMENTOS DE VASO DA MADEIRA DE Carapa guianensis Aulb. EM DUAS FITOFISIONOMIAS DA AMAZÔNIA
}

\section{ECOLOGICAL ANATOMY OF THE VESSEL ELEMENTS IN THE WOOD OF Carapa guianensis Aulb. IN TWO AMAZON VEGETATION TYPES}

\author{
Mateus Ferreira Lima'; Karem Santos da Silva²; Maila Janaína Coêlho de Souza ${ }^{3}$; Marcelo \\ Mendes Braga Júnior ${ }^{4}$; Luiz Eduardo de Lima Melo ${ }^{5}$
}

DOI: https://doi.org/10.31692/978-65-991061-7-0.93-101

\section{RESUMO}

Os estudos de anatomia ecológica são realizados frequentemente para obter informações sobre as estratégias anatômicas dos vegetais em diferentes ambientes. Este estudo teve como objetivo identificar as estratégias anatômicas adaptativas do xilema secundário da espécie Carapa guianensis Aulb. ocorrente em duas fitofisionomias da Amazônia: mata de planície de inundação (várzea) e mata de terra firme. As coletas foram realizadas no estado do Pará, em mata de terra firme no município de Nova Ipixuna e em mata de várzea no município de Belém. Foram coletadas amostras do lenho a 1,30 m do solo por método não destrutivo de dez indivíduos em cada fitofisionomia e retiradas as medições de DAP (diâmetro à altura do peito) e altura das árvores. Após a coleta foram feitos corpos de provas para as análises microscópicas. A caracterização anatômica microscópica qualitativa e quantitativa foi realizada através da montagem de lâminas histológicas permanentes e material macerado para as contagens e mensurações dos caracteres anatômicos, de acordo com as normas da IAWA. Para os resultados quantitativos foi aplicado teste de homogeneidade de variância (teste Bartlett a 5\% de significância) e análise de variância (ANAVA) a 5\% de significância. A partir das análises microscópicas foi constatado que apenas a frequência dos vasos diferiu estaticamente entre os dois ambientes e que os indivíduos de terra firme apresentam uma eficiência maior na condução hídrica de acordo com as características analisadas. As diferenças anatômicas observadas neste estudo são devido a alguns fatores, como: altitude, latitude e longitude dos locais, indicando a influência do meio sobre essas características.

Palavras-Chave: Adaptabilidade, Xeromórfica, Mesomórfica.

\begin{abstract}
Ecological anatomy studies are often performed to obtain information about the anatomical strategies of plants in different environments. This study aimed to identify the adaptive anatomical strategies of the secondary xylem of the species Carapa guianensis Aulb. occurring in two Amazon vegetation types: floodplain forest and unflooded terra firme forest. Assuming that the unflooded environment has a higher degree of xeromorphism. The samples were collected in the state of Pará, in a unflooded terra firme forest in Nova Ipixuna municipality and in the floodplain forest of the municipality of Belém. Samples were collected at 1,30 $\mathrm{m}$ from the soil by non-destructive method of ten trees and the measurements of DBH (diameter at breast height) and height of the trees were taken. After the collection,

1 Graduando em Engenharia Florestal, Universidade do Estado do Pará, Mateus ferreiralima@ outlook.com

2 Graduada em Engenharia Florestal, Universidade do Estado do Pará, karem.monaliza@ gmail.com

3 Mestranda em Ciências Florestais, Universidade Federal do Rio Grande do Norte, mailajcsouza@gmail.com

4 Graduando em Engenharia Florestal, Universidade do Estado do Pará, bragajuniorm@ gmail.com

5 Professor Dr. em Ciência e tecnologia da Madeira, Universidade do Estado do Pará, Luizeduardo.limamelo@gmail.com
\end{abstract}


specimen were made for the microscopic analysis. The qualitative and quantitative microscopic anatomical characterization was performed according to IAWA standards. For the quantitative results a analysis of variance (ANAVA) at 5\% significance was applied. From the microscopic analysis it was verified that only the frequency of the vessels differed statically between the two environments and that the individuals presented a greater efficiency in the water conduction according to the characteristics analyzed. The anatomical differences observed in this study are due to some factors, such as: altitude, latitude and longitude of the sites, indicating the influence of it on these characteristics.

Keywords: Adaptability, Xeromorphic, Mesomorphic.

\section{INTRODUÇÃO}

O domínio fitogeográfico amazônico possui diferentes fitofisionomias, dentre elas, a floresta de terra firme e planícies inundáveis, conhecida regionalmente como várzea. Relacionando florestas de várzea e mata de terra firme, há na mata de terra firme uma biodiversidade de espécies animais e vegetais mais expressiva, o que pode ser explicado pela quantidade reduzida de espécies que possuem capacidade de se adaptar a esses ambientes que apresentam sazonalidade de inundação (GAMA et al., 2005). Segundo Scudeller (2009) a floresta de terra firme apresenta fisionomia de biomassa abundante e geralmente o seu subbosque é aberto, o solo dessa área é considerado pobre em nutrientes, sendo a maior parte da matéria orgânica proveniente das plantas.

A anatomia ecológica busca estudar os elementos estruturais anatômicos da madeira, e os mesmos podem passar por adaptações de acordo com o ambiente onde o vegetal está inserido. Em espécies lenhosas, as variações ambientais se refletem na estrutura anatômica da madeira provocando alterações de caráter morfológico e estrutural de caráter seletivo, os quais levam inicialmente indivíduos e por fim espécies a se adaptarem (BAAS E CARLQUIST, 1985; BARAJAS-MORALES, 1985; ALVES E ANGYALOSSY-ALFONSO, 2000, 2002), essa plasticidade é principalmente observada nas características anatômicas quantitativas, sendo algumas já relatadas e fortemente aceitas como adaptações específicas de determinados ambientes. Vasos mais numerosos e de menor diâmetro tangencial, elementos de vasos mais curtos e pontoações intervasculares com aberturas menores são adaptações aceitas como sendo estratégias do sistema condutor para aperfeiçoar o transporte de água, voltadas para uma condução segura no sistema solo-planta-atmosfera, pois maiores tensões dentro de elementos vasculares estreitos os torna menos vulneráveis ao embolismo, e estes são considerados como aspectos anatômicos xeromórficos (CARLQUIST, 1966; BARAJASMORALES, 1985). Assim vasos de maior diâmetro tangencial e menos frequentes estão voltadas para uma condução hidráulica eficiente e são característicos de ambientes com boa disponibilidade de água no solo, chamados de mésicos. Deste modo, a segurança mostra-se 
como valor adaptativo em condições xéricas e a eficiência como valor adaptativo em condições mésicas (ZIMMERMANN, 1983).

Gonçalves et al. (2009) chama atenção para a boa plasticidade fisiológica que a espécie Carapa guianensis Aubl. possui quando a mesma é submetida ao estresse hídrico, fato que permite seu estabelecimento em solos com diferentes disponibilidades de água, desde que não sejam muito secos. Baseando-se no exposto, o presente estudo visa contribuir para um melhor entendimento das diferenças anatômicas referentes a vasos (frequência, diâmetro e comprimento) no xilema secundário da espécie Carapa guianensis Aubl. quando a mesma é submetida a dois ambientes diferentes. Tendo como objetivo comprovar que essas diferenças ocorrem, quando a espécie está inserida em duas fitofisionomias da região Amazônica: ambiente de várzea e terra firme.

\section{FUNDAMENTAÇÃO TEÓRICA}

\section{Gênero Carapa}

A família Meliaceae é formada por 51 gêneros, e possui aproximadamente 1.400 espécies (RAUBER, 2010). O gênero Carapa, se destaca pela sua importância econômica, o mesmo, tem ocorrência na África, América Central e América do Sul (AUBLET, 1977; CANDOLLE, 1878; LOUREIRO et al., 1979). A espécie C. guianensis, especificamente, ocorre no domínio fitogeográfico designado amazônico com distribuição nas regiões Norte, nos estados do Pará, Acre, Amazonas e Amapá, e na região Nordeste, no estado do Maranhão, segundo o Flora do Brasil, do jardim botânico do Rio de Janeiro.

Caracterização das áreas de estudo

O domínio fitogeográfico amazônico possui uma grande variedade de fitofisionomias. Coutinho (2006) afirma que o domínio fitogeográfico amazônico é composto por várias fitofisionomias como a floresta de terra firme formada pelos igapós, as caatingas do rio negro, os campos rupestres, floresta tropical pluvial do Zonobioma I, etc.

Os solos de terra firme, são predominantemente classificados em latossolos amarelos ou vermelhos. Hopkins (2005) diferencia terra firme como uma floresta que se encontra em ambiente que não sofre inundações periódicas, terreno mais elevado e com grande biodiversidade. Segundo este autor as florestas de terra firme podem ser classificadas ainda quanto à composição do solo e relevo em: florestas de platô, baixio e campinarana.

As áreas de planícies inundáveis ou áreas de várzea são indiscutivelmente um complexo ecossistema, apresenta uma imensurável riqueza biológica, os rios pertencentes ao domínio fitogeográfico amazônico juntamente com suas áreas inundáveis compõem uma 
cobertura de mais de $300.000 \mathrm{~km}^{2}$. Segundo Benatti et al. (2005) juridicamente não há um consenso entre pesquisadores sobre um conceito delimitante sobre o que é floresta de várzea, entretanto a lei 12.651 de 25 de maio de 2012 conceitua em seu capítulo $1^{\circ}$ artigo terceiro, áreas de várzea como áreas marginais a cursos de água sujeitas a enchentes e inundações periódicas. Vieira e Santos (1987) definem os solos, quase sempre argilosos, como adubados pelas chuvas periódicas que promovem inundações constantes, possuem grande riqueza de húmus devido à lixiviação da floresta, são originários de terrenos sedimentares (terciários e quaternários), classificados como hidromorfos e argiloarenoso.

Para estar presente em dois ambientes marcadamente distintos as plantas possuem diferentes formas de adaptação, alterações na anatomia do lenho, principalmente relacionadas as características dos elementos de vasos, responsáveis pela condução de água e sais minerais do solo até as folhas. Nesse contexto a anatomia ecológica do lenho é definida como um conjunto de adaptações pelas quais os elementos estruturais anatômicos da madeira passam a manifestar em virtude do ambiente onde o vegetal está inserido, dentre as células do lenho, os elementos de vaso são marcadamente influenciados pelo ambiente, em ambientes secos por exemplo, o câmbio produz vasos pequenos e em maior quantidade pois vasos estreitos são mais seguros em períodos de seca, por serem mais estreitos é muito mais difícil ocorrer embolias no vegetal.

Luchi (2004), em sua pesquisa sobre o comportamento da espécie Croton urucurana Baill em três ambientes diferentes, apresentou diferenças significativas quanto aos caracteres anatômicos do lenho destas espécies principalmente nos ambientes com níveis de umidade muito divergentes. A autora apresenta valores de maior diâmetro de vasos, maior frequência de vasos solitários, maior diâmetro das fibras e diâmetro de lúmen das fibras, menor largura de raio e raio de parênquima axial e maior comprimento de fibras, nos ambientes com maior umidade em comparação ao ambiente com menor umidade.

\section{METODOLOGIA}

As coletas foram realizadas em duas áreas de floresta nativa no estado do Pará: Ilha do Combú, localizada no município de Belém, mesorregião metropolitana de Belém, correspondente à área de várzea (VZ); a segunda coleta foi realizada numa área de projeto de Assentamento Agroextrativista intitulado Praialta Piranheira, localizada no município de Nova Ipixuna no sudeste do estado do Pará, correspondente à área de terra firme (TF). 
Tabela 1: Comparação entre as áreas estudadas Fonte: (OLIVEIRA, 2008ª); (Plano Diretor do município de Nova Ipixuna, 2006 $\left.{ }^{\mathrm{b}}\right)$; (JARDIM, 2000 $)$; (MENEZES, 2002 ${ }^{\mathrm{d}}$ ); (http://pt.mygeoposition.com ${ }^{\mathrm{e}}$ ).

\begin{tabular}{lll}
\hline Descrição das áreas & VZ & TF \\
\hline Altitude (m) & $14^{\mathrm{e}}$ & $118^{\mathrm{b}}$ \\
Latitude (W) & $1^{\circ} 25^{\prime \mathrm{c}}$ & $4^{\mathrm{o}} 43^{\prime \mathrm{d}}$ \\
Longitude (S) & $48^{\circ} 25^{\mathrm{c}^{\mathrm{c}}}$ & $49^{\circ} 14^{\prime \mathrm{d}}$ \\
Precipitação anual $(\mathbf{m m})$ & $2.500^{\mathrm{c}}$ & $2.000 / 2.250^{\mathrm{a}}$ \\
Temperatura média $\left({ }^{\circ} \mathbf{C}\right)$ & $27^{\mathrm{c}}$ & $26^{\mathrm{a}}$ \\
Altura médias das árvores coletadas $(\mathbf{m})$ & 22,10 & 19,55 \\
\hline
\end{tabular}

Figura 1: Áreas de coleta do lenho da espécie Carapa guianensis Aubl. Legenda: a) Terra firme; b) Várzea. Fonte: Própria

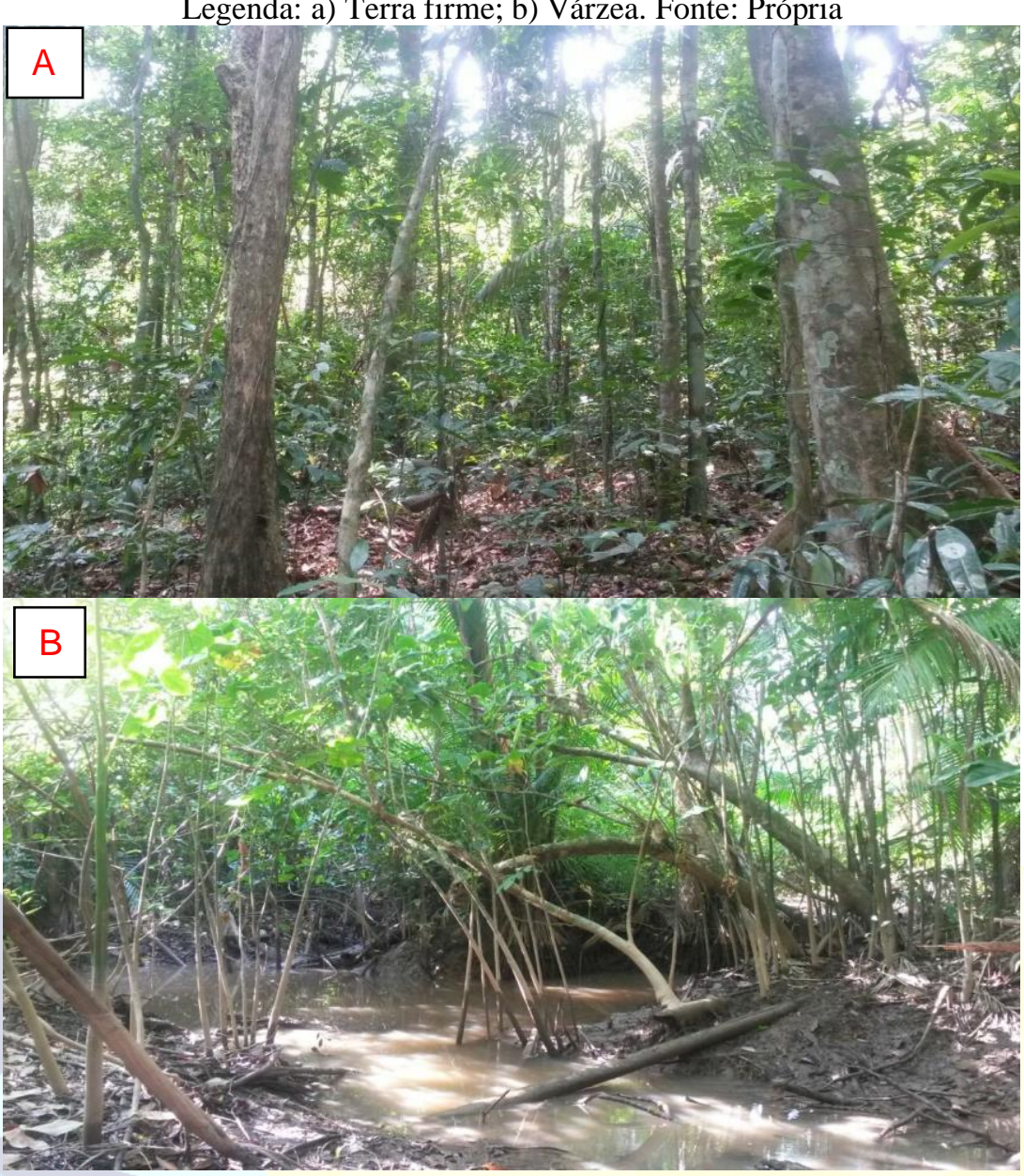

Foram selecionados aleatoriamente dez indivíduos adultos em cada área de coleta de Terra Firme e Várzea. Em seguida foram retiradas amostras do caule a 1,30 m do solo, contendo floema, câmbio e xilema, as amostras foram obtidas por meio de método nãodestrutivo: incisões paralelas feitas com serrote ou facão e a total remoção com martelo e formão, após a remoção das amostras foi aplicado tinta comercial antifúngica e antimicrobiana na injúria realizada no caule.

Após a coleta das amostras de madeira, estas foram reduzidas a corpos de prova com 
dimensão de 1,5 cm de aresta, e devidamente orientados nos três planos convencionais de estudo da anatomia do lenho (transversal, longitudinal tangencial e longitudinal radial).

Após a preparação dos corpos de prova foram feitos cortes, em micrótomo de deslize (Leitz 1208) com o objetivo de confeccionar lâminas histológicas. Para cada indivíduo foram montadas dez lâminas histológicas, cada uma contendo os planos transversais, tangenciais e radiais, e cinco lâminas de material macerado por indivíduo. Para a mensuração dos elementos anatômicos dissociados foi preparada maceração a partir da solução de Franklin (1945) o material já macerado foi corado com safranina $1 \%$ e etanol $50 \%$ e foram montadas as lâminas em glicerina diluída em água destilada (glicerina 50\%).

Após a montagem das lâminas foram realizadas as análises em microscopia de luz transmitida, foram analisados os parâmetros quantitativos: comprimento dos elementos de vasos $(\mu \mathrm{m})$, diâmetro dos vasos $(\mu \mathrm{m})$, frequência de vasos $\left(\mathrm{n}^{\mathrm{o}}\right.$ de vasos $\left./ \mathrm{mm} 2\right)$ e índice de agrupamento dos vasos ( ${ }^{\circ}$ de vasos/grupo). As mensurações foram realizadas seguindo as recomendações do IAWA Committee (1989). Os parâmetros anatômicos qualitativos foram também analisados segundo as recomendações da IAWA Committee (1989).

Quanto a análise estatística, foi realizado de forma preliminar o teste de homogeneidade de variância (teste Bartlett a 5\% de significância) para todas as características da madeira e aplicada análise de variância (ANAVA) nos dados obtidos. Como o estudo envolve apenas um fator (espécie) e este possui apenas 1 grau de liberdade (dois níveis - dois ambientes diferentes), o teste $\mathrm{F}$ é equivalente a qualquer tipo de teste de média, ou seja, o resultado apresentado pelo Teste $\mathrm{F}$ da análise de variância a $5 \%$ de significância é suficiente para testar as hipóteses:

* Hipótese nula (H0): as médias populacionais são iguais.

* Hipótese alternativa (H1): as médias populacionais são diferentes, ou seja, pelo menos uma das médias é diferente das demais.

\section{RESULTADOS E DISCUSSÃO}

Qualitativamente não foram observadas diferenças nas características anatômicas da madeira de $C$. guianensis entre os ambientes avaliados, assim procedeu-se a descrição geral das características anatômicas para espécie.

Características anatômicas microscópicas: Lenho com camadas de crescimento distintas, demarcadas por parênquima marginal com frequentes canais intercelulares axiais de origem traumática, e espessamento diferencial da parede das fibras no lenho tardio. Vasos/poros com porosidade difusa, agrupados em solitários e múltiplos radiais, o 
agrupamento dos vasos solitários foi de $40,77 \%$ e 59,22\% de múltiplos, sendo múltiplos de 2 e 3 bem representativos, e ocorrência de até múltiplos de 8, o arranjo dos vasos segue o padrão diagonal e radial, apresentam contorno oval a circular, placa de perfuração simples (figura 2c), pontuações guarnecidas ausentes, pontuações intervasculares alternas, pontuações radiovasculares com aréolas distintas em tamanho e forma no raio da célula (figura $2 \mathrm{a}-\mathrm{b}$ ). Fibras predominantemente libriformes com pontuações simples ou areoladas diminutas septadas e não septadas. Parênquima axial em faixas marginais, dividido em cinco a oito células por strand de parênquima. Raios heterogêneos, formados por células procumbentes principalmente com 2 a 4 filas de células marginais quadradas e eretas, sem estratificação, raios obstruídos por substância de cor escura. Cristais prismáticos presentes nas células quadradas e/ou eretas. Canais intercelulares axiais de origem traumática.

A presença de camadas de crescimento distintas, vasos solitários e múltiplos, com porosidade difusa, placa de perfuração simples, parênquima marginal, raios não estratificados e formados por células procumbentes foram também descritas para a espécie por outros autores (DÜNISCH et al., 2002; TRIVIZOR, 2011; ALBUQUERQUE, 2012; CORANDIN et al., 2010; SANTINI JÚNIOR, 2013).

Os vasos do lenho de C. guianensis nas áreas de terra firme e de várzea na região Amazônica são pouco abundantes (IAWA Committee 1989). Segundo Wheller et al. (2007) porosidade difusa são comuns em madeiras das regiões da América do Sul, porém arranjo diagonais são mais comuns em madeiras com porosidade em anéis do que em madeiras com porosidade difusa. Segundo o mesmo autor as placas de perfuração simples proporcionam vantagens hidráulicas na maioria dos ambientes, e pontuações intervasculares alternas são mais comuns em regiões tropicais e possuem uma estrutura mais forte do que as pontuações dispostas horizontalmente.

Figura 2: Características microscópicas dos poros/vasoso no xilema secundário da espécie Carapa guianensis. Legenda: a) seção tangencial contendo pontoações simples ou areoladas (seta); b) seção tangencial contendo pontoações simples ou areoladas (seta); (C) placa de perfuração simples (seta). Escala de barras: $50 \mu \mathrm{m}$. Fonte:

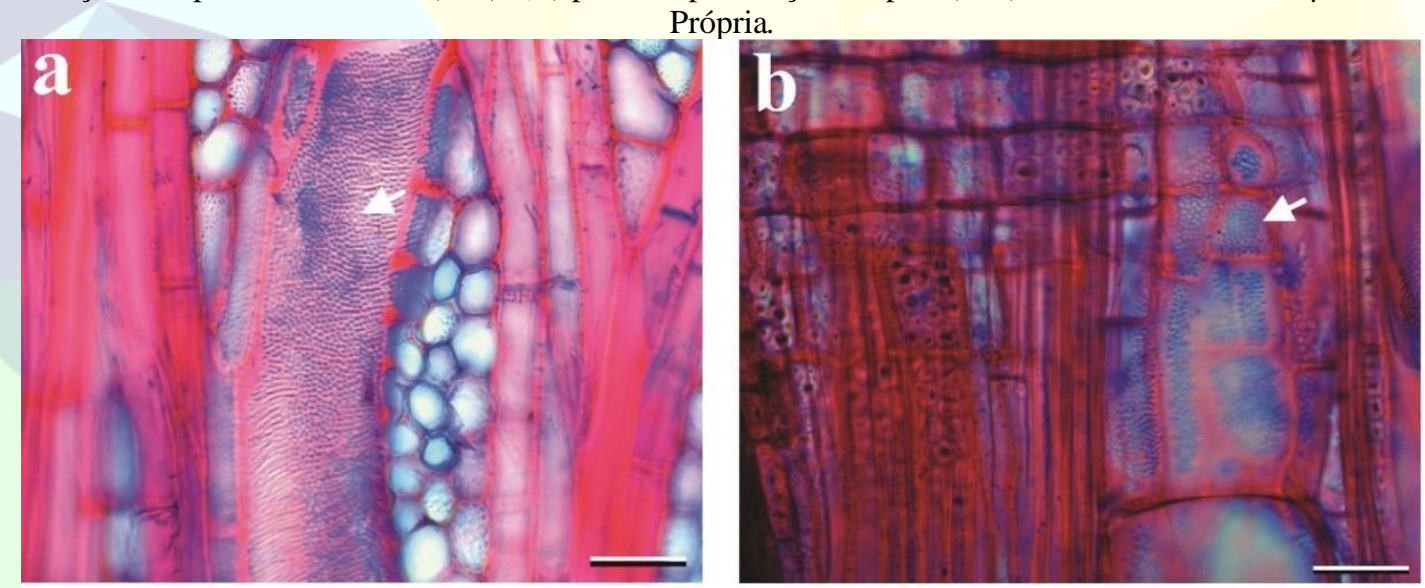




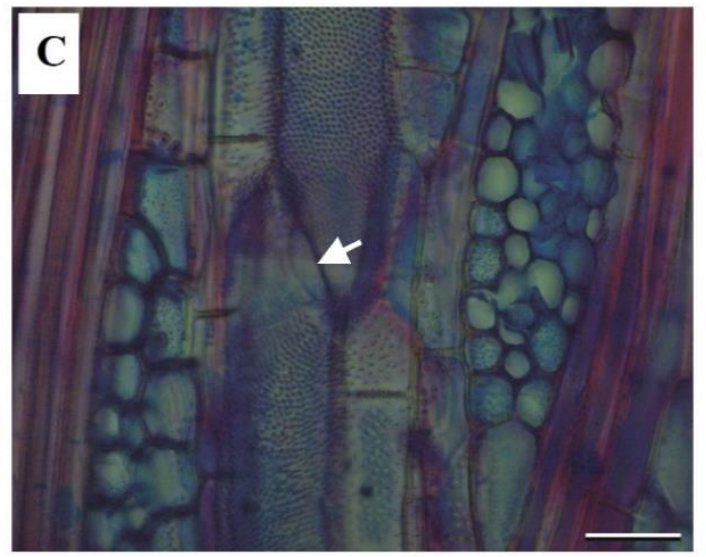

A Tabela 2 demonstra os valores médios relacionados às características morfométricas dos vasos nas madeiras analisadas, assim como a influência da fitofisionomia sobre os tais elementos anatômicos.

Tabela 2: Dados quantitativos de C. guianensis nas áreas de terra firme e de várzea na região Amazônica. Legenda: C.V. $(\%)=$ Coeficiente de variação. Letras diferentes (a ou b) indicam que houveram diferenças estáticas significantes, de acordo com o teste F. Fonte: Própria.

\begin{tabular}{llll}
\hline Características anatômicas quantitativas & Terra Firme & Várzea & C.V.(\%) \\
\hline Frequência dos vasos $\left(\mathrm{n} / \mathrm{mm}^{2}\right)$ & $9,20 \mathrm{~b}$ & $11,43 \mathrm{a}$ & 43,24 \\
Diâmetro dos vasos $(\mu \mathrm{m})$ & $162,82 \mathrm{a}$ & $160,94 \mathrm{a}$ & 15,85 \\
Comprimento dos vasos $(\mu \mathrm{m})$ & $419,41 \mathrm{a}$ & $408,74 \mathrm{a}$ & 21,83 \\
\hline
\end{tabular}

Figura 3: Características microscópicas comparando caracteres anatômicos da espécie Carapa guianensis nos ambientes de Terra firme e várzea. Legenda: a e b) frequência de vasos. Barra de escala: 200 m. Fonte: Própria.
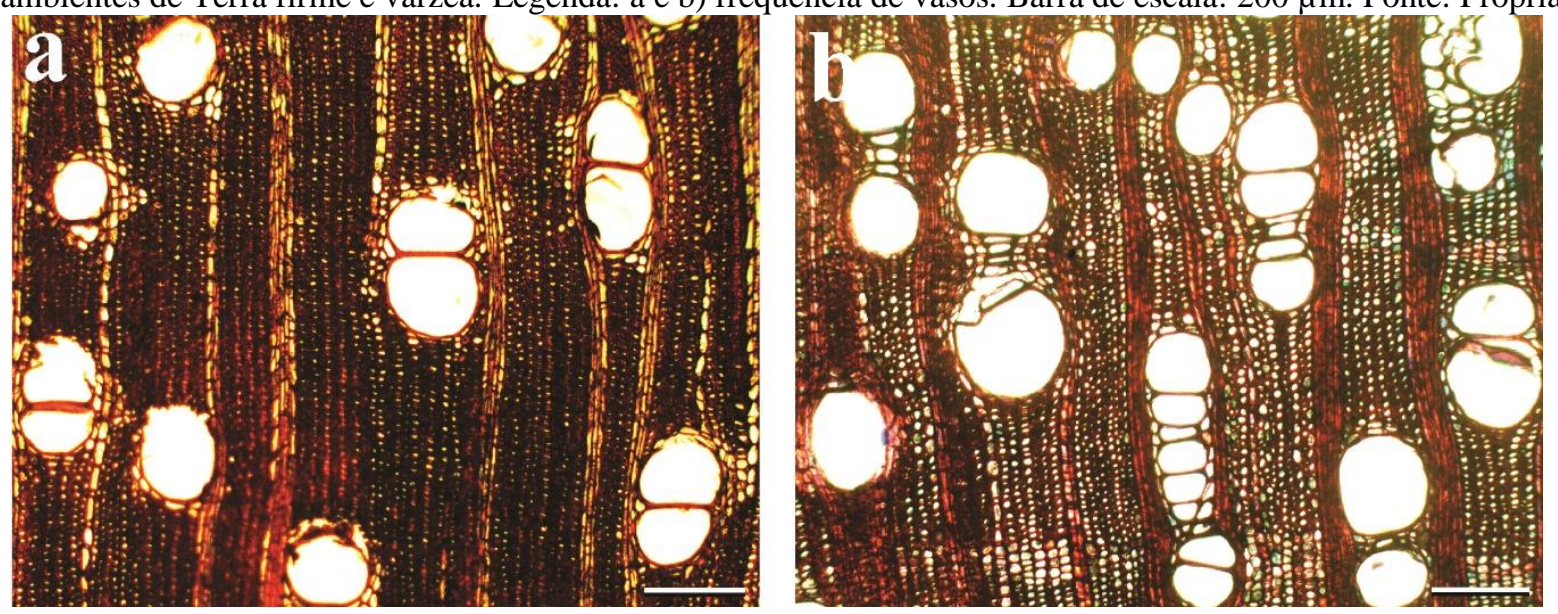

Quantitativamente foi observado o efeito estatisticamente significativo, teste $\mathrm{F}$ a $5 \%$ de significância, somente para a frequência de vasos por $\mathrm{mm}^{2}$ (Tabela 2). Na área de várzea ocorreu maior frequência de vasos, porém não houve variação em relação ao diâmetro e comprimento dos vasos entre as duas áreas de estudo. Trivizor (2011) analisando a mesma espécie obteve para comprimento de vasos um valor médio de $499 \mu \mathrm{m}$. Santini Júnior (2013) em seu estudo de descrição anatômica de C. guianensis observou que o diâmetro dos vasos variou de 104-202 $\mu \mathrm{m}$, no presente trabalho os valores médios foram de 160,94 $\mu \mathrm{m}$ e 162,82 
$\mu \mathrm{m}$.

Segundo Micco e Aronne (2002) muitas espécies vegetais atuam de modo a encontrar um equilíbrio entre segurança e eficiência na condução hídrica, a adaptação dos vegetais a ambientes com diferentes disponibilidades hídrica pode ocorrer por meio de uma combinação da mudança dos elementos anatômicos, uma característica pode servir de compensação à outra. As características morfológicas e morfométricas dos indivíduos analisados provavelmente contribuem para a permanência e propagação da espécie estudada, assim o conhecimento do processo de condução hídrica permite o entendimento da dinâmica de ocupação territorial da espécie.

\section{CONCLUSÕES}

Os indivíduos da espécie Carapa guianensis pertencentes à terra firme e à várzea diferiram em relação à anatomia do lenho. Confirmando a hipótese em que se baseou esta pesquisa, a frequência dos vasos apresentou média significativamente diferente entre os dois ambientes, corroborando a ideia de que os indivíduos possuem características adaptadas para seu local de inserção.

Os indivíduos apresentam características adaptativas em decorrência do ambiente onde estão inseridas em virtude das variáveis ambientais, como latitude, longitude, altitude, disponibilidade hídrica.

\section{REFERÊNCIAS}

ALBUQUERQUE, A. R. Anatomia comparada do lenho e do carvão aplicada na identificação de 76 espécies da floresta Amazônica, no estado do Pará, Brasil. Dissertação (Mestrado). Escola Superior de Agricultura “Luiz de Queiroz”. Piracicaba, 2012. 249p.

ALVES, E. S. \& V. ANGYALOSSY-ALFONSO. Ecological trends in the wood anatomy of some Brazilian species. 1. Growth rings and vessels. IAWA Journal, v. 21, p.3-30, 2000.

ALVES, E. S. \& V. ANGYALOSSY-ALFONSO. Ecological trends in the wood anatomy of some Brazilian species. 1. Axial parenchyma, rays and fibres. IAWA Journal, v.23, p.391418, 2002.

AUBLET, F. Histoire des plantes de la Guiana française. J. Cremer, Germany. Vol.1 Supl. 32-34. 1977.

BAAS, P. \& S. CARLQUIST. A comparison of the ecological wood anatomy of the floras of Southern California and Israel. IAWA Journal. v. 6, p. 349-353, 1985.

BARAJAS-MORALES, J. Wood structural differences between trees of two tropical forests in Mexico. IAWA Bulletin n. s. v. 6, p. 355-364, 1985. 
BENATTI , J. H.; SURGIK, A. C. S.; TRECCANI, G. D.; MCGRATH , D. G.; GAMA, A. S. P. da. A questão fundiária e o manejo dos recursos naturais da várzea: análise para a elaboração de novos modelos jurídicos. Edições Ibama / Pro Várzea. AM, 2005.

BRASIL, Novo Código Florestal. Lei n 12.651, de 25 de maio de 2012.

CANDOLle, C. DE. Meliaceae. In: A. \& C. DE CANDOLLE. Monographiae Phanerogarum. 1878

CARLQUIST, S. Wood anatomy of Compositae: a summary, with comments on factors controlling wood evolution. Aliso, v. 6, p. 25-44, 1966.

CORADIN, V. T. R.; CAMARGOS, J. A. A.; PASTORE, T. C. M.; CHRISTO, A. G. Madeiras comerciais do Brasil: chave interativa de identificação baseada em caracteres gerais e macroscópicos $=$ Brazilian commercial timbers:interactive identification key based on general and macroscopic features. Serviço Florestal Brasileiro, Laboratório de Produtos Florestais: Brasília, 2010.CD-ROM.

COUTINHO, L. M. (2006). O Conceito de Bioma. Acta botânica brasílica. 2006, 13-23.

DÜNISCH, O.; BAUCH, J.; GASPAROTTO, L. Formation of increment zones and intraannual growth dynamics in the xylem of Swietenia macrophylla, Carapa guianensis, and Cedrela odorata (Meliaceae). Iawa Journal, v. 23, n. 2, p. 101-119, 2002.

FRANKLIN, G. L. Preparation of thin sections of synthetic resins and wood-resin composites, and a new macerating method for wood. Nature, London, v. 155, n. 3924, p. 51$51,1945$.

GAMA, J. R. V.; SOUZA, A. L.; MARTINS, S. V.; SOUZA, D. R. Comparação entre Florestas de Várzea e de Terra Firme do Estado do Pará. Revista Árvore. MG, v.29, n.4, p.607-616, 2005.

HOPKINS, M. J. G. Flora da Reserva Ducke, Amazonas, Brasil. Rodriguésia. PA, 2005.

IAWA COMMITTEE. List of microscope features for hardwood identification. IAWA Bull. New Ser., v. 10, n. 3, p. 234-332, 1989.

LOUREIRO, A. A.; SILVA, M. F. DA; ALENCAR, J. DA C. Essências madeireiras da Amazônia. INPA/SUFRAMA, 1979, 187 p. v. 2.

LUCHI, A. E. Anatomia do lenhoff de Croton rucurana Baill. (Euphorbiaceae) de solos com diferentes níveis de umidade. Revista Brasil. 2004. 27.2: 271-280.

MICCO, V. de; ARONNE, G. Plant resposnses to Drought stress from morphological to molecular features. Springer. 2002.

RAUBER, R. C. Dendroecologia de Cedrela fissilis Vell. (Meliaceae) em um ecótono de florestas subtropicais montanas do Brasil. 80f. 2010. Tese de Doutorado. Dissertação (M estrado em Ecologia) - Universidade Federal do Rio Grande do Sul, Porto Alegre. 
SANTINI JUNIOR, L. Descrição macroscópica e microscópica da madeira aplicada na identificação das principais espécies comercializadas no estado de São PauloProgramas (Doctoral dissertation). SP, 2013.

SCUDELLER, V. V.; RAMOS, R. A.; CRUZ, M. E. G. Flora fanerogâmica da floresta de terra-firme na RDS Tupé. Biotupé: meio físico, diversidade biológica e sociocultural do baixo Rio Negro, Amazônia Central. (EN Santos-Silva, VV Scudeller, eds.). Universidade Estadual do Amazonas-UEA Ltda., Manaus, v. 2, p. 109-120, 2009.

TRIVIZOR, T. T. Anatomia comparada do lenho de 64 espécies arbóreas de ocorrência natural na floresta tropical amazônica no estado do Pará. Dissertação (Mestrado). Escola Superior de Agricultura "Luiz de Queiroz". Piracicaba, 2011.214p.

VIEIRA, L. S.; SANTOS, P. C. T. C. dos. Amazônia seus Solos e Outros Recursos Naturais. SP, 1987.

ZIMMERMANN, M.H. Xylem structure and ascent sap. Berlin: Springer Verlag, 1983 